\title{
Israeli National Policy for Carbapenem-Resistant Enterobacteriaceae Screening, Carrier Isolation and Discontinuation of Isolation
}

\author{
Ester Solter, RN; ${ }^{1, a}$ Amos Adler, MD; ${ }^{1,2, a}$ Bina Rubinovitch, MD; ${ }^{1}$ Elizabeth Temkin, DrPH; ${ }^{1}$ David Schwartz, $\mathrm{PhD} ;{ }^{1}$ \\ Debby Ben-David, MD; ${ }^{1}$ Samira Masarwa, RN; ${ }^{1}$ Yehuda Carmeli, MD, MPH; ${ }^{1,2}$ Mitchell J. Schwaber, MD, MSc ${ }^{1,2}$
}

\begin{abstract}
Aвstract. Since 2006, Israel has been confronting an outbreak of carbapenem-resistant Enterobacteriaceae (CRE), and in 2007 Israel implemented a national strategy to contain spread. The intervention was initially directed toward acute-care hospitals and later expanded to include an established reservoir of carriage in long-term-care hospitals. It included regular reporting of CRE cases to a central registry and daily oversight of management of the outbreak at the institutional level. Microbiological methodologies were standardized in clinical laboratories nationwide. Uniform requirements for carrier screening and isolation were established, and a protocol for discontinuation of carrier status was formulated. In response to the evolving epidemiology of CRE in Israel and the continued need for uniform guidelines for carrier detection and isolation, the Ministry of Health in 2016 issued a regulatory circular updating the requirements for CRE screening, laboratory diagnosis, molecular characterization, and carrier isolation, as well as reporting and discontinuation of isolation in healthcare institutions nationwide. The principal elements of the circular are contained herein.
\end{abstract}

Infect Control Hosp Epidemiol 2018;39:85-89

Since 2006, Israel has been confronting an outbreak of carbapenem-resistant Enterobacteriaceae (CRE). In 2007, Israel implemented a national strategy to contain its spread. ${ }^{1,2}$ At the time of implementation of the national intervention, more than 1,000 patients were known to be infected, and it is estimated that more than 10,000 were colonized. ${ }^{3}$ The intervention was initially directed toward acute-care hospitals and was later expanded to include an established reservoir of carriage in long-term-care hospitals. ${ }^{4}$

The intervention included regular reporting of CRE cases to a central registry and daily oversight of management of the outbreak at the institutional level (Supplementary Figure 1). To accomplish comprehensive detection of cases, microbiological methodologies were standardized in clinical laboratories nationwide. In addition, uniform requirements for carrier screening and isolation were established, directed at carbapenemaseproducing Enterobacteriaceae (CPE), and implementation was overseen daily. The accumulation of knowledge and experience enabled the differentiation of isolation requirements according to patient population ${ }^{5}$ and the formulation of a protocol for discontinuation of carrier status.

During the initial 5 years of the outbreak, the predominant CRE strain was Klebsiella pneumoniae clonal complex (CC) 258 , and KPC was practically the exclusive carbapenemase. ${ }^{6}$
During the past several years, the epidemiology of CRE in Israel has evolved, with a marked reduction in incidence of CC258, the appearance of additional carbapenemases (primarily NDM and OXA-48) and increasing predominance of non-carbapenemase-producing CRE. Among the reasons for this shift in epidemiology are the successful containment of spread of CC258 as well as medical tourism, humanitarian aid provided by Israeli hospitals, and global trends. ${ }^{7,8}$

In response to the evolving epidemiology of CRE in Israel and the continued need for uniform guidelines for carrier detection and isolation, the Ministry of Health $(\mathrm{MOH})$ issued a regulatory circular updating the requirements for CRE screening, laboratory diagnosis, molecular characterization, and carrier isolation, as well as reporting and discontinuation of isolation in healthcare institutions nationwide. The principal elements of the circular, issued in 2016, are contained herein.

\section{Guidelines for Preventing the Spread of CRE}

"Carbapenem-resistant Enterobacteriaceae" is defined as any Enterobacteriaceae isolate not susceptible to meropenem (MIC $>1 \mu \mathrm{g} / \mathrm{mL}$ or disk diffusion diameter $<23 \mathrm{~mm}$ ) or having a carbapenemase-coding gene. A standardized protocol for microbiological identification and molecular characterization of CRE

\footnotetext{
Affiliations: 1. National Center for Infection Control, Israel Ministry of Health; 2. Sackler Faculty of Medicine, Tel Aviv University.

${ }^{a}$ Authors of equal contribution.

Received September 7, 2017; accepted September 14, 2017; electronically published December 15, 2017

(c) 2017 by The Society for Healthcare Epidemiology of America. All rights reserved. 0899-823X/2018/3901-0012. DOI: 10.1017/ice.2017.211
} 

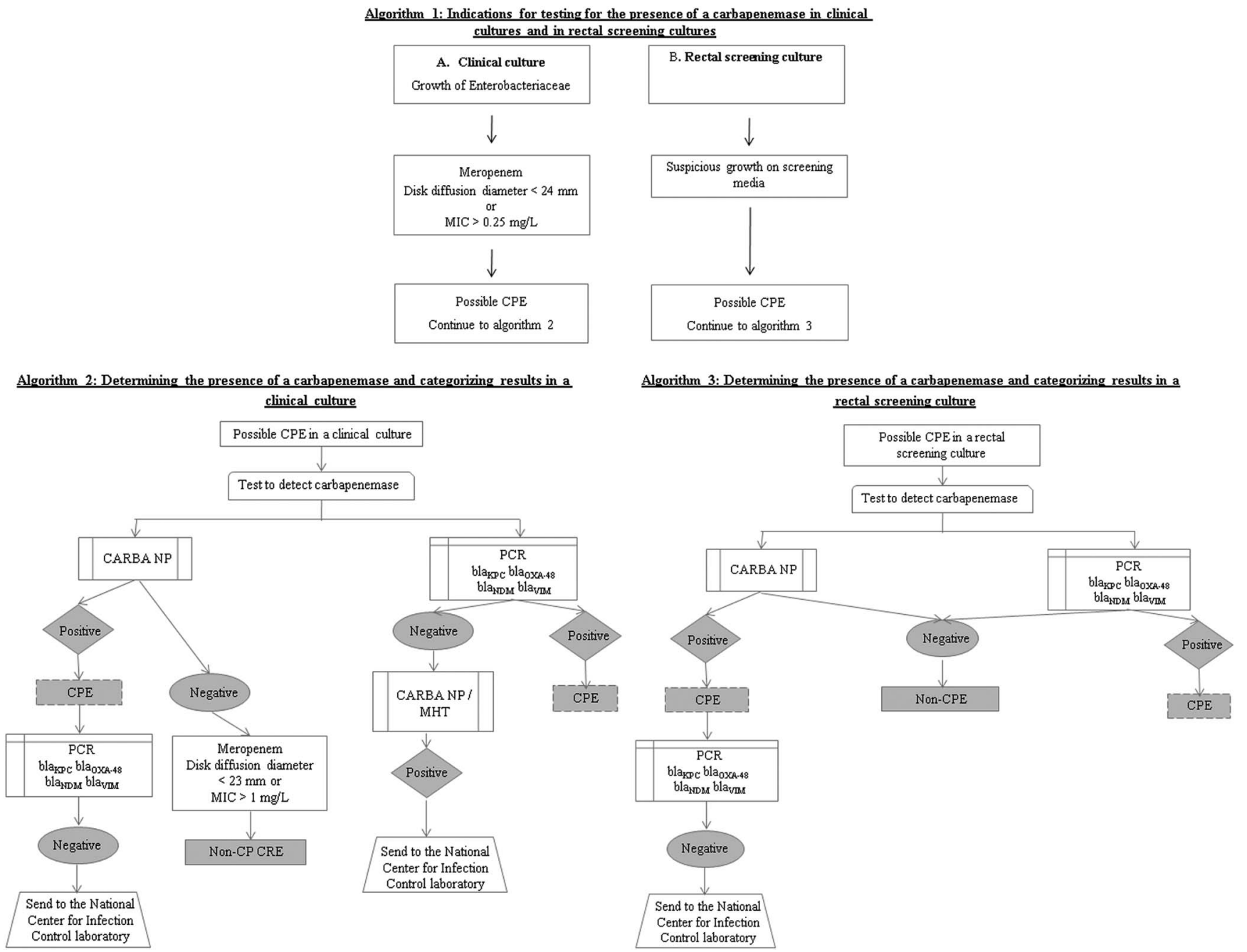

FIGURE 1. Algorithms for performing carbapenemase tests. 1) Indications for testing; 2) Carbapenemase tests for isolates from clinical specimens; 3) Carbapenemase tests for isolates from screening specimens.

isolated from clinical and screening cultures has been issued. This protocol leads to the determination of which CRE isolates contain a carbapenemase-producing gene (carbapenemase-producing Enterobacteriaceae, CPE). Algorithms guiding the workup of suspected CRE isolates in clinical and screening cultures are presented in Figure 1. Because of the heightened public health threat posed by CPE as compared with non-carbapenemase-producing CRE (non-CP CRE), national infection control efforts are focused on preventing the spread of CPE.

The infection prevention staff in the hospital is responsible for identifying contacts. Timely identification of $\mathrm{CPE}$ carriers is pivotal to the success of interventions aimed at preventing spread, when linked to effective isolation measures. Therefore, populations at risk of carriage, by recent contact with a carrier or an endemic environment, must be screened as early as possible. Early screening carries the risk of falsenegative results in patients with recent $\mathrm{CPE}$ acquisition. Therefore, infection prevention staff in each facility should consider the need for repeat screening if the initial screening is negative.

Populations at risk of carriage by recent contact with a carrier. Contacts include patients who share a room with a newly identified carrier or were cared for by the same staff (eg, same primary nurse), as well as other patients at high risk of being colonized and/or infected by the new carrier, according to individual staff judgment. Screening should be carried out within 24 hours of the detection of carriage in the index patient. Whenever a new carrier is detected by contact screening, all of the patient's contacts should also be screened, according to the same criteria applied in the initial round of screening. Contacts who have since been transferred to other wards in the hospital should be located and screened.

In certain circumstances, the entire ward should be screened following detection of a new CPE case. These include (1) wards with a CPE cohort area and an area for noncarriers; (2) high-risk units (eg, intensive care and/or step-down units, 
TA B LE 1. Israeli National Guidelines for the Care of Patients with Carbapenem-Resistant Enterobacteriaceae in Acute-Care Versus PostAcute-Care Hospitals ${ }^{\mathrm{a}}$

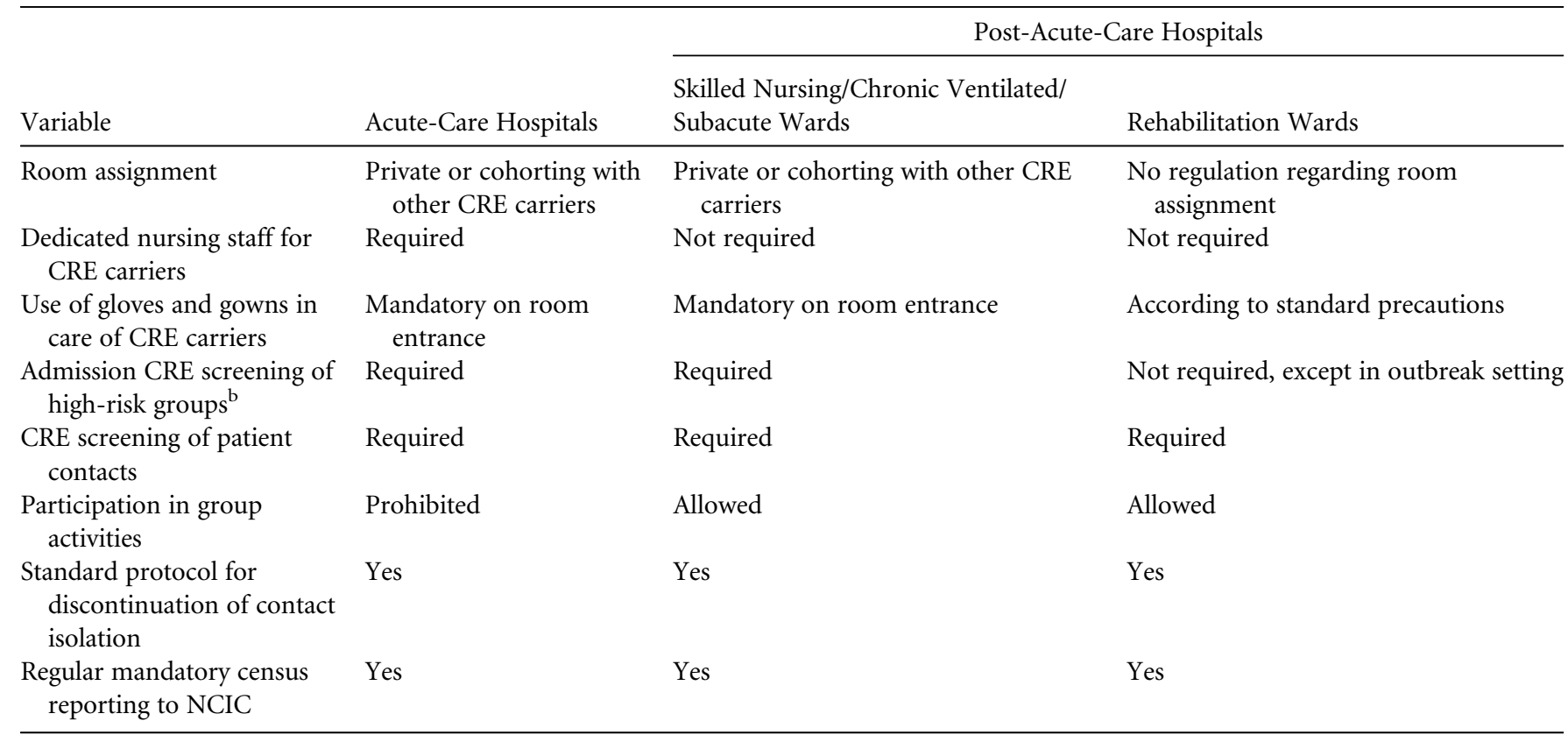

NOTE. CRE, carbapenem-resistant Enterobacteriaceae; NCIC, National Center for Infection Control.

${ }^{\text {a}}$ Reproduced from Ben-David et al. ${ }^{5}$

${ }^{\mathrm{b}}$ High-risk groups were defined as patients transferred from other facilities or patients with earlier hospitalization within the previous 6 months.

hematology-oncology, bone marrow transplant, solid organ transplant, pediatrics, and preterm newborns); and (3) any unit in which, during screening, a high prevalence of carriage is discovered.

Populations at risk of carriage by recent contact with an endemic environment. The infection prevention staff in each hospital should set a policy for screening populations at risk for carriage. Specific policies must be set for screening patients upon hospital admission and/or in units with a high risk of cross-transmission or morbidity, according to the hospital's data, including the following populations: (1) patients admitted directly from hospitals in other countries or who were treated in hospitals in other countries (to be screened upon admission); (2) patients admitted directly from other hospitals or institutions, including nursing homes, or patients who resided in a hospital or other healthcare facility in the past 6 months (to be screened upon admission); and (3) patients who were transferred within the hospital from a high-risk unit (to be screened upon transfer). Policies must also address the management of screened patients (ie, hospitalization conditions such as preemptive isolation) from the time of screening until results are received, and of patients whose preliminary culture results are positive.

\section{Hospitalization Conditions for CPE Carriers in General Hospitals}

Carbapenemase-producing Enterobacteriaceae carriers should be placed under contact isolation precautions in a delineated area clearly separated from other patients. This area should be supplied with equipment dedicated for use in this CPE cohort area only. Nursing staff assigned to care for CPE carriers should not be assigned care duties outside the CPE cohort area during the same shift. The entry of other staff and visitors to this area should be supervised to ensure strict adherence to required protocols for gowning, gloving, removal of gowns and gloves and hand hygiene. Carriers of CPE should be separated by room and equipment within the cohort area according to carbapenemase produced to limit mixing of reservoirs of different genotypes of CPE. Carriers of non-CP CRE should be maintained on contact precautions but not within the CPE cohort area.

Required hospitalization conditions for CPE carriers in long-term-care facilities (LTCFs) are less stringent than those for carriers in acute-care hospitals, and these vary according to patient population based on relative risk of cross-transmission. These conditions have been described elsewhere, and are summarized in Table $1 .{ }^{5}$

\section{Discontinuation of CPE Carrier Status in Known Carriers}

Detection of CPE has significant implications for patients because it necessitates isolation throughout their hospitalization. CPE carriage may resolve spontaneously, but in most cases it is likely to last $>1$ month. ${ }^{9-11}$ While it is in a carrier's interest to be reassessed for continued carriage and removed from isolation if testing is negative, premature removal from 
isolation (ie, during the period when the patient is at ongoing risk of carriage) may result in cross transmission of CPE. Therefore, it is necessary to understand the causes of prolonged carriage, which lower the chances that CPE carrier status will be discontinued, and to define appropriate methods for discontinuing carrier status.

Most available data on duration of carriage and sensitivity of detection methods refer to KPC-producing Klebsiella pneumoniae. ${ }^{5,9-11}$ Extrapolation to other CPE should be done with caution. There is considerable variability among carriers, with many factors potentially influencing duration of carriage. The following general guidelines aim to help staff make informed choices about the process of discontinuing carrier status.

Among the factors that increase the risk of ongoing CPE carriage are living in a long-term-care facility, poor functional status, residence on a ward on which the incidence of CPE acquisition is high, previous antibiotic therapy and $<3$ months elapsed since the last positive CPE culture. Given the obvious importance of location (general hospital vs longterm-care facility; healthcare facility vs home), the following recommendations are divided accordingly:

1. General guidelines for the process of discontinuing carrier status

1.1. The duration of carriage is $>1$ month in most cases. Therefore, negative results within $<1$ month after a positive culture may reflect insufficient test sensitivity.

1.2. As a rule, $\mathrm{CPE}$ testing for the purpose of discontinuing carrier status should not be undertaken $<1$ month after the positive culture.

1.3. The infection prevention staff in each facility is responsible for making decisions about individual cases and for setting the general policy regarding discontinuation of carrier status.

2. Selecting patients for discontinuation of carrier status in the community

2.1. Patients who live permanently at home, especially those who have good functional status and do not require repeated hospitalization, have a high likelihood of resolution of CPE carriage.

2.2. The outpatient healthcare provider should commence the process of discontinuing carrier status 3 months after the last positive culture.

3. Selecting patients for discontinuation of carrier status in general hospitals

3.1. Discontinuing carrier status during a hospitalization in which CPE carriage is documented is generally not recommended.

3.2. Among readmitted carriers, the discontinuation process should be undertaken immediately upon hospital admission, if the patient resides in the community and at least 3 months have passed since the last positive culture.

3.3. Candidates for discontinuation of carrier status should be cared for under contact precautions, in a private room, outside of the CPE cohort area, until test results are available.

4. Selecting patients for discontinuation of carrier status in LTCFs

4.1. This population is at highest risk for prolonged carriage and for having a repeat positive test following the discontinuation process. At the same time, this is the most significant population of carriers that stand to benefit from discontinuation of carrier status, and in most cases, repeat carriage is not documented.

4.2. It is recommended to begin the discontinuation process in all patients hospitalized in LTCFs provided at least 3 months have passed since the last positive culture.

4.3. In patients with many risk factors for prolonged carriage, including poor functional/physical status (eg, chronically ventilated patients), frequent antibiotic treatment, and hospitalization in a ward with a high prevalence of carriage, consider avoiding or delaying the start of the discontinuation process beyond the defined time frame of 3 months.

4.4. It is important to carry out the discontinuation process fully and to conduct follow-up testing for recrudescence of detectable carriage after the process is completed.

\section{Process and Methods for Discontinuation of Carrier Status}

In determining the process for discontinuing carrier status, it is important to consider the intermittent nature of carriage detection, ${ }^{10}$ which is also influenced by the sensitivity of different testing methods. Therefore, we require 3 consecutive negative tests: 2 screening cultures and 1 polymerase chain reaction (PCR) test for the relevant carbapenemase-encoding gene. This protocol, which has greater sensitivity than culture alone, is required to minimize false-negative results in a population with a high pretest probability of continued carriage. $^{12,13}$

If CPE was isolated from a clinical (ie, nonscreening) site in which carriage is likely (eg, urine, respiratory tract, wound), then at least once during the discontinuation process a culture should be taken from the same site, if relevant (eg, there is no need to repeat a culture from a wound that has healed). The laboratory should be notified that the culture is for the purposes of CPE screening.

The discontinuation process should be carried out over at least 1 week (ie, a span of 7 days between the first test and the third), to optimize the likelihood that a successfully completed process truly represents absence of carriage (unpublished data). 


\section{Follow-Up after Completion of the Discontinuation Process}

Patients with presumed eradication of CRE carriage have been found to have a $13 \%$ chance of reverting to carrier status, either by recrudescence of prior carriage at again detectable levels, or by reinfection ${ }^{14,15}$. Therefore, a test for CPE carriage should be repeated upon each new admission to a healthcare facility.

For patients at high risk of carriage recurrence, or when the patient is hospitalized in a ward in which recurrence is liable to have severe consequences, testing for carriage should be repeated periodically.

\section{Information Transfer}

An effective CPE containment strategy requires accurate and timely dissemination of information. Therefore, a standard terminology has been designated (see Figure 1). CPE carriage status should be reported in every hospitalization discharge summary and letter of transfer to another institution, as well as to the Ministry of Health. ${ }^{2}$ In patients whose carrier status has been discontinued, the discontinuation of carrier status should be noted in the discharge summary or transfer letter, and to the Ministry of Health, and should become a permanent part of the patient's medical record. The infection prevention staff in each facility is responsible for making decisions about individual cases. A nationwide, web-based program to facilitate seamless data entry and transfer to relevant providers, and to limit our present reliance on specific, limited personnel is under development.

In conclusion, the guidelines summarized in this document have been developed over a decade of confronting the nationwide CPE outbreak in Israel, and they reflect the epidemiology of the Israeli outbreak and the structure and function of the Israeli healthcare system. Our strategy has been successful in containing the outbreak. We believe that the principles outlined here provide a framework for other healthcare systems confronting $\mathrm{CPE}$. While a centralized approach is necessary for effective CPE control, differences between countries in terms of available resources and healthcare system structures, as well as variability in the biology and dynamic epidemiology of the CPE strains and enzymes, will require adaptation and ongoing assessment of our strategy's suitability to locally prevailing conditions.

\section{ACKNOW LEDGMENTS}

Financial support: No financial support was provided relevant to this article.

Potential conflicts of interest: All authors report no conflicts of interest relevant to this article.

Address correspondence to Mitchell J. Schwaber, MD, MSc, Director, National Center for Infection Control, Israel Ministry of Health, 6 Weizmann St, Tel Aviv 6423906 Israel (mitchells@tlvmc.gov.il).

SUPPLEMENTARY MATERIAL

To view supplementary material for this article, please visit https://doi.org/10.1017/ice.2017.211.

\section{REFERENCES}

1. Schwaber MJ, Lev B, Israeli A, et al. Containment of a countrywide outbreak of carbapenem-resistant Klebsiella pneumoniae in Israeli hospitals via a nationally implemented intervention. Clin Infect Dis 2011;52:848-855.

2. Schwaber MJ, Carmeli Y. An ongoing national intervention to contain the spread of carbapenem-resistant Enterobacteriaceae. Clin Infect Dis 2014;58:697-703.

3. Schwaber MJ, Carmeli Y. The impact of a carbapenem-resistant Enterobacteriaceae outbreak on facilitating development of a national infrastructure for infection control in Israel. Clin Infect Dis 2017;65:2144-2149.

4. Ben-David D, Masarwa S, Navon-Venezia S, et al. Carbapenemresistant Klebsiella pneumoniae in post-acute-care facilities in Israel. Infect Control Hosp Epidemiol 2011;32:845-853.

5. Ben-David D, Masarwa S, Adler A, Mishali H, Carmeli Y, Schwaber MJ. A national intervention to prevent the spread of carbapenem-resistant Enterobacteriaceae in Israeli post-acute care hospitals. Infect Control Hosp Epidemiol 2014; 35:802-809.

6. Navon-Venezia S, Leavitt A, Schwaber MJ, et al. First report on a hyperepidemic clone of KPC-3-producing Klebsiella pneumoniae in Israel genetically related to a strain causing outbreaks in the United States. Antimicrob Agents Chemother 2009;53:818-820.

7. Adler A, Shklyar M, Schwaber MJ, et al. Introduction of OXA-48-producing Enterobacteriaceae to Israeli hospitals by medical tourism. J Antimicrob Chemother 2011;66:2763-2766.

8. Lerner A, Solter E, Rachi E, et al. Detection and characterization of carbapenemase-producing Enterobacteriaceae in wounded Syrian patients admitted to hospitals in northern Israel. Eur J Clin Microbiol Infect Dis 2016;35:149-154.

9. Schechner V, Kotlovsky T, Tarabeia J, et al. Predictors of rectal carriage of carbapenem-resistant Enterobacteriaceae (CRE) among patients with known CRE carriage at their next hospital encounter. Infect Control Hosp Epidemiol 2011;32:497-503.

10. Feldman N, Adler A, Molshatzki N, et al. Gastrointestinal colonization by KPC-producing Klebsiella pneumoniae following hospital discharge: duration of carriage and risk factors for persistent carriage. Clin Microbiol Infect 2013;19:E190-E196.

11. Bar-Yoseph H, Hussein K, Braun E, Paul M. Natural history and decolonization strategies for ESBL/carbapenem-resistant Enterobacteriaceae carriage: systematic review and meta-analysis. J Antimicrob Chemother 2016;71:2729-2739.

12. Schechner V, Straus-Robinson K, Schwartz D, et al. Evaluation of PCR-based testing for surveillance of KPC-producing carbapenem-resistant members of the Enterobacteriaceae family. J Clin Microbiol 2009;47:3261-3265.

13. Hindiyeh M, Smollen G, Grossman Z, et al. Rapid detection of $b l a_{\mathrm{KPC}}$ carbapenemase genes by real-time PCR. J Clin Microbiol 2008;46:2879-2883.

14. Bart Y, Paul M, Eluk O, Geffen Y, Rabino G, Hussein K. Risk factors for recurrence of carbapenem-resistant Enterobacteriaceae carriage: case-control study. Infect Control Hosp Epidemiol 2015;36:936-941.

15. Adler A, Lifshitz Z, Gordon M, et al. Evolution and dissemination of the Klebsiella pneumoniae clonal group 258 throughout Israeli post-acute care hospitals, 2008-13. J Antimicrob Chemother 2017;72:2219-2224. 